\title{
空气分级炉膛煤粉燃烬模型及实炉试验对比
}

\author{
刘福国 ${ }^{1,2}$ 郭新根 ${ }^{1,2}$ \\ (1. 国网山东省电力公司电力科学研究院 济南 250002; \\ 2. 山东电力研究院 济南 250002)
}

\begin{abstract}
摘要: 传统的炉膛分区段传热设计模型忽略了煤粉燃烬计算, 适用于非空气分级燃烧。随着空气分级低 $\mathrm{NO}_{x}$ 燃烧技术的普遍 应用, 在炉膛分区段传热计算中引入煤粉燃烧模型以确定沿炉膛高度燃烬分布, 对于提高炉膛上部屏式或辐射受热面蒸汽温 度设计准确性有较为重要的意义。建立了改进型分区段传热计算和煤粉燃烧相耦合的空气分级炉膛燃烬和传热模型, 对一台 空气分级低 $\mathrm{NO}_{x}$ 燃烧锅炉进行了全负荷工况试验, 采用该模型对试验工况进行燃烬和传热模拟, 得到空气分级锅炉炉膛煤粉 燃烧过程的物理图景以及煤粉沿炉膛高度燃尽分布, 并研究了燃烧模式和表面反应动力学参数等对燃烬度分布的影响。结果 表明, 炉膛出口煤粉颗粒燃烬度数值解与大部分测试数据吻合较好, 煤粉颗粒燃烧后期灰分引起热退火抑制效应以及炉内局 部烟气含氧量分布不均匀是引起模型误差的主要因素, 所建立的燃烧和传热耦合模型与传统的炉膛分区段传热模型计算量相 当, 适合工程应用。
\end{abstract}

关键词: 电厂; 锅炉; 空气分级; 煤粉燃烧; 模型; 燃烬

中图分类号: TK22

\section{Pulverized Coal Burnout Determination in Furnace with Air Staging and It's Comparison with Field Test}

\author{
LIU Fuguo ${ }^{1,2}$ GUO Xingen ${ }^{1,2}$
}

(1. State Grid Shandong Electric Power Research Institute, Jinan 250002;

2. Shandong Electric Power Research Institute, Jinan 250002)

\begin{abstract}
The traditional zone-by-zone computational approach neglecting particles burnout calculation in furnace heat transfer design is applicable to non air staged combustion. With the extensive air staged low $\mathrm{NO}_{x}$ combustion technology, advanced coal combustion model integrated with the heat transfer approach to determine the burnout distribution along the furnace height is of great significance to improve the design accuracy of steam temperature in platen super heater or radiant reheater in the upper zone of the furnace. An improved zone-by-zone computational approach incorporated with a more detailed coal combustion model is developed to determine burnout rate of bituminous coal in tangentially fired furnace with air staging, field tests is carried out in the case boiler covering the regular range of variation of operating loads. The tested furnace conditions are used as inputs for the zone-by-zone computational approach coupled with combustion model to predict coal burnout, results of the simulation provide a clear insight into the burnout processes in air staging combustion. The effects of combustion mode and surface reaction kinetics parameters on burnout are also investigated. Results from the simulations are compared against the measured values, showing a reasonable agreement in most test scenarios. Thermal annealing, ash inhibition and inhomogeneous distribution of oxygen content in flue gas are the main cause of the model error. The computational complexity in the incorporated burnout model are equivalent to that of the traditional zone-by-zone computational approach, and suitable for engineering application.
\end{abstract}

Key words: power plant; boiler; air staging; coal combustion; modeling; burnout

\section{0 前言}

锅炉燃煤经过破碎和研磨形成煤粉, 被空气气 流携带喷入炉膛, 在数秒内穿过炉膛而完成燃烧过
程。在锅炉运行中，常用飞灰可燃物含量表示煤粉 燃烬程度, 是重要的监控参数。飞灰中未燃烬碳损 失降低了锅炉效率, 增加燃料消耗 ${ }^{[1-2]}$, 且飞灰作为 水泥和混凝土的添加剂, 可燃物含量过高会降低水 泥的抗冻融性，从而影响飞灰的无害化利用，增加 环境压力 ${ }^{[3-4]}$ 。

20200201 收到初稿, 20200715 收到修改稿 
近年来, 随着炉膛空气分级低 $\mathrm{NO}_{x}$ 燃烧技术的 普遍应用, 电厂锅炉降低飞灰可燃物含量面临新的 压力和挑战。对于传统的非空气分级燃烧, 在主燃 烧区一次性供给所有燃烧空气，煤粉在主燃烧区燃 烬度达 $95 \%$ 以上 ${ }^{[5]}$, 炉内煤粉持续处于有氧气氛中, 在炉膛各区段中, 煤粉燃烬分布主要决定于煤的燃 烧特性, 采用传统方法对炉膛进行传热设计时, 煤 粉未燃烬损失以及沿炉膛高度燃烬度分布通常根据 设计煤种选用经验数据 ${ }^{[5]}$ 。但是, 对于空气分级低 $\mathrm{NO}_{x}$ 燃烧锅炉, 从主燃烧区送入的空气量通常低于 理论空气量, 剩余空气从分离燃烬风区(Separated over fire air, SOFA)送入, 形成分级燃烧 ${ }^{[-7]}$, 煤粉 在主燃烧区燃烬度由该区供给的空气量决定, 对于 烟煤锅炉, 该空气量只有理论空气量的 $70 \%$ 左右, 在主燃烧区大部分空间内, 烟气中氧气被消耗殆尽, 燃烧处于停滞状态, 与传统燃烧方式相比, 这部分 缺氧的炉膛空间未能得到有效利用, 因此锅炉在进 行空气分级低 $\mathrm{NO}_{x}$ 燃烧技术改造后, 飞灰可燃物含 量升高, 锅炉效率下降。

空气分级低 $\mathrm{NO}_{x}$ 燃烧系统改造后, 锅炉煤粉燃 烬度下降不是目前面临的唯一问题。在炉膛传热设 计时, 合理确定煤粉沿炉膛高度燃烬分布对提高锅 炉汽温设计准确性有较为重要意义, 现代大容量锅 炉, 炉膛上部布置大量的辐射或半辐射受热面, 空 气分级送风改变了煤粉沿炉膛高度燃烬度分布, 造 成火焰中心升高, 从而引起炉膛上部受热面吸热量 的变化, 很多锅炉进行空气分级低 $\mathrm{NO}_{x}$ 燃烧技术改 造后, 出现蒸汽超温或欠温现象 ${ }^{[8-9]}$, 主要原因是对 煤粉沿炉膛高度燃烬度分布变化估计不足或过量引 起的。

近年来, 煤粉燃烧过程的应用研究得到快速发 展, 积累了大量煤焦反应动力学参数的经验数据。 菲尔德等 ${ }^{[10-11]}$ 认为煤焦颗粒的燃烧包括氧气传输到 颗粒表面, 以及颗粒表面发生化学反应等两个主要 过程, 给出了包含表面反应动力项和扩散项的单个 颗粒燃烧方程; HURT 等 ${ }^{[12-13]}$ 提出了焦碳燃烬动力 学(Carbon burnout kinetic, CBK)模型, 把颗粒燃烧后 期灰分对剩余碳燃烬的热退火抑制效应加入到单颗 粒氧化模型; STEPHENSON ${ }^{[14]}$ 基于 CBK 模型开发 了计算机程序, 根据锅炉实际运行参数和燃用煤质 特性预测煤粉燃烬度, 用于优化锅炉运行操作, 提 高煤粉燃烬度。

分区段传热模型是经过长期工程应用检验的炉 膛设计和校核模型 ${ }^{[5]}$, 但其主要缺陷是没有进行煤 粉燃烬计算, 随着空气分级 $\mathrm{NO}_{x}$ 燃烧技术的普遍应
用, 在炉膛分区段传热计算中引入煤粉燃烧计算以 确定沿炉膛高度燃烬分布已成为迫切需要, 在一台 空气分级低 $\mathrm{NO}_{x}$ 燃烧锅炉上, 对煤粉燃烧模型和分 区段传热模型进行耦合求解, 并将煤粉燃烬度求解 结果和实际测试数据进行了对比。

\section{1 炉膛煤粉燃烧模型}

\section{1 煤粉粒度分布}

燃烧模型是针对具有一定粒径分布的煤粉颗 粒。使用不同孔径的煤粉笁篎分煤粉, 可得到煤粉 颗粒分布规律, 采用篎孔为 $x_{\mathrm{j}}$ 的煤粉耖, 篮上煤粉 的百分比为 $R_{x j}, R_{x j}$ 随 $x_{\mathrm{j}}$ 的变化符合 Rosin-Rammler 公式 ${ }^{[15]}$

$$
R_{x j}=100 \exp \left(-b x_{\mathrm{j}}^{n}\right)
$$

只要测定 200、90 $\mu \mathrm{m}$ 两个孔径的篮分数据, 即煤粉细度 $R_{90}$ 和 $R_{200}$ 的值, 根据式(2)、(3)可确定 式(1)中的系数 $b$ 和指数 $n$, 从而确定煤粉粒径分布。

$$
\begin{gathered}
n=\frac{\lg \ln \left(100 / R_{200}\right)-\lg \ln \left(100 / R_{90}\right)}{\lg (200 / 90)} \\
b=\ln \left(100 / R_{90}\right) / 90^{n}
\end{gathered}
$$

\section{2 挥发分析出和燃烧}

煤的干燥主要在磨煤机内完成, 假设 $1 \mathrm{~kg}$ 收到 基煤, 干燥掉 $M_{\mathrm{ar}} / 100 \mathrm{~kg}$ 水分后, 煤粉颗粒由固定 碳、挥发分和灰分组成, 共 $1-M_{\mathrm{ar}} / 100 \mathrm{~kg}$ 。

煤粉在炉膛内析出挥发分数量与煤质成分、炉 膛温度有关, 对于 $1 \mathrm{~kg}$ 收到基煤产生的煤粉, 析出 的挥发分数量 $V_{\mathrm{f}}$ 为 ${ }^{[16]}$

$$
\begin{gathered}
V_{\mathrm{f}}=k\left[1-\exp \left(-D I \times T_{\mathrm{p}} / T_{\mathrm{st}}\right)\right] \\
D I=0.0219 V_{\mathrm{d}}-0.208
\end{gathered}
$$

式中, $V_{\mathrm{f}}$ 为 $1 \mathrm{~kg}$ 收到基煤在炉内最终析出的挥发分 量, $\mathrm{kg} ; k$ 为煤干燥无灰基换算到收到基的系数, $\left(100-A_{\mathrm{ar}}-M_{\mathrm{ar}}\right) / 100 ; D I$ 为脱挥发分指数; $T_{\mathrm{p}}$ 为 颗粒热解温度, $\mathrm{K} ; T_{\mathrm{st}}$ 为实验室进行煤工业分析的 标准温度, $T_{\mathrm{st}}=1223 \mathrm{~K} ; A_{\mathrm{ar}}$ 为煤的收到基灰分含 量, $\% ; M_{\mathrm{ar}}$ 为煤的收到基水分含量, $\% ; V_{\mathrm{d}}$ 为实验 室分析的煤干燥基挥发分, \%。

假设挥发分析出后瞬间燃尽, 挥发分析出和燃 烧符合一阶反应模型 ${ }^{[17]}$

$$
\begin{gathered}
\mathrm{d} V / \mathrm{d} t=-k_{\mathrm{v}} V \\
k_{\mathrm{v}}=A_{\mathrm{v}} \exp \left(-E_{\mathrm{v}} / R T_{\mathrm{p}}\right)
\end{gathered}
$$


在挥发分析出的初始时刻, 满足

$$
t=0, \quad V=V_{\mathrm{f}}
$$

式中, $V$ 为燃烧过程 $t$ 时刻颗粒中剩余的挥发分, $\mathrm{kg} ; t$ 为时间, $\mathrm{s} ; k_{\mathrm{v}}$ 为脱挥发分速率系数; $A_{\mathrm{v}}$ 为频 率因子, $\mathrm{s}^{-1} ; E_{\mathrm{v}}$ 为活化能, $\mathrm{J} / \mathrm{mol} ; R$ 为理想气体常 数, $R=8.314 \mathrm{~J} \cdot \mathrm{mol}^{-1} \cdot \mathrm{K}^{-1}$ 。

式(7)中频率因子 $A_{\mathrm{v}}$ 、活化能 $E_{\mathrm{v}}$ 与煤的性质有 关, 按下式计算 ${ }^{[16]}$

$$
\begin{gathered}
E_{\mathrm{v}}=30400 O_{\mathrm{daf}} / C_{\mathrm{daf}}+24600 \\
\ln \left(A_{\mathrm{v}}\right)=0.105 E_{\mathrm{v}}+3.42
\end{gathered}
$$

式中, $O_{\mathrm{daf}}$ 为煤的干燥无灰基氧含量, $\%$; $O_{\mathrm{daf}}$ 为干 燥无灰基碳含量, \%;

\section{3 煤焦颗粒燃烧}

在炉膛环境下, 根据式(4), $1 \mathrm{~kg}$ 收到基煤析出 挥发分 $V_{\mathrm{f}}(\mathrm{kg})$, 则产生煤焦量 $C_{\mathrm{f}}(\mathrm{kg})$ 为

$$
C_{\mathrm{f}}=1-A_{\mathrm{ar}} / 100-M_{\mathrm{ar}} / 100-V_{\mathrm{f}}
$$

为进行单个颗粒燃烧计算, 采用 13 个不连续的 粒径组分 $x_{j}=3,7,12,22,33,48,67,90,123$, $168,213,255,350 \mu \mathrm{m}$ 表示式(1)的煤粉颗粒分 布 ${ }^{[10]}$, 直径为 $x_{j}(j=1 \sim 13)$ 的煤粉颗粒的质量份额 $w_{j}$ 可按式(12)计算

$$
w_{j}=\exp \left(-b x_{j-1}^{n}\right)-\exp \left(-b x_{j}^{n}\right)
$$

式中, $x_{j-1}$ 为与粒径 $x_{j}$ 相邻的小粒径组分的直径, $\mu \mathrm{m}$ 。

假设所有颗粒组分在干燥和热解过程中, 按相 同的比例析出水分和挥发分，且粒径以同一倍数 $\delta$ 进行膨胀, 热解结束得到第 $j$ 个组分的煤焦颗粒的 初始直径 $D_{\mathrm{p}, j}^{0}(\mathrm{~m})$ 为

$$
D_{\mathrm{p}, j}^{0}=\delta x_{j} \times 10^{-6}
$$

假设煤焦颗粒完全是由碳组成, $1 \mathrm{~kg}$ 收到基煤 产生的煤焦颗粒中，第 $j$ 个组分颗粒的个数为

$$
N_{j}=\frac{6 w_{j} C_{\mathrm{f}}}{\rho_{\mathrm{p}}^{0} \pi\left(D_{\mathrm{p}, j}^{0}\right)^{3}}
$$

式中, $\rho_{\mathrm{p}}^{0}$ 为煤焦颗粒的初始密度, $\mathrm{kg} / \mathrm{m}^{3}$ 。

第 $j$ 个组分单个颗粒的质量 $(\mathrm{kg})$ 为

$$
m_{\mathrm{c} j}^{0}=\rho_{\mathrm{p}}^{0} \pi\left(D_{\mathrm{p}, j}^{0}\right)^{3} / 6
$$

根据球形颗粒燃烧模型, 煤焦颗粒碳的消耗速 率与表面反应速度、氧气扩散速度决定, 第 $j$ 个组 分的单个颗粒的碳消耗速率为 ${ }^{[18-19]}$

$$
\frac{\mathrm{d} m_{\mathrm{c} j}}{\mathrm{~d} t}=-\pi D_{\mathrm{p}, j}^{2}\left(R_{\mathrm{diff}}^{-1}+R_{\mathrm{c}}^{-1}\right)^{-1} P_{\mathrm{O}_{2}}
$$

$$
\begin{gathered}
R_{\mathrm{c}}=A_{\mathrm{f}} \exp \left(-E_{\mathrm{a}} / R T_{\mathrm{p}}\right) \\
R_{\text {diff }}=\frac{2 \varphi D_{0} M_{\mathrm{c}}}{R T_{0} D_{\mathrm{p}, \mathrm{j}}}\left(\frac{T_{\mathrm{p}}+T_{\mathrm{g}}}{2 T_{0}}\right)^{0.75}
\end{gathered}
$$

在煤焦燃烧的初始时刻 $t_{\mathrm{c}}$, 满足

$$
t=t_{\mathrm{c}}, \quad m_{\mathrm{c} j}=m_{\mathrm{c} j}^{0}
$$

式中, $m_{\mathrm{c} j}$ 为第 $j$ 个组分煤焦颗粒质量, $\mathrm{kg} ; D_{\mathrm{p}, \mathrm{j}}$ 为 第 $j$ 个组分煤焦颗粒直径, $\mathrm{m} ; R_{\mathrm{diff}}$ 为扩散反应速率 系数, $\mathrm{kg} /\left(\mathrm{m}^{2} \cdot \mathrm{s} \cdot \mathrm{Pa}\right) ; R_{\mathrm{c}}$ 为表面化学反应速率系 数, $\mathrm{kg} /\left(\mathrm{m}^{2} \cdot \mathrm{s} \cdot \mathrm{Pa}\right) ; P_{\mathrm{O}_{2}}$ 为气流中氧气分压力, $\mathrm{Pa}$; $A_{\mathrm{f}}$ 为表面反应频率因子, $\mathrm{kg} /\left(\mathrm{m}^{2} \cdot \mathrm{s} \cdot \mathrm{Pa}\right) ; E_{\mathrm{a}}$ 为煤 焦颗粒反应活化能, $\mathrm{J} / \mathrm{mol} ; T_{\mathrm{p}}$ 为煤焦颗粒温度, $\mathrm{K}$; $\varphi$ 为反应机理因子 (产物为 $\mathrm{CO}_{2}$ 时, $\varphi=1$; 产物为 $\mathrm{CO}$ 时, $\varphi=2) ; D_{0}$ 为在参考温度 $T_{0}$ 下, 氮氧系统的 二元扩散系数, $D_{0}=3.49 \times 10^{-4} \mathrm{~m}^{2} / \mathrm{s} ; T_{0}$ 为参考温度, $T_{0}=1600 \mathrm{~K} ; M_{\mathrm{c}}$ 为碳的摩尔质量, $M_{\mathrm{C}}=$ $0.012 \mathrm{~kg} / \mathrm{mol} ; T_{\mathrm{g}}$ 为气体温度, $\mathrm{K}$ 。

煤焦颗粒燃烧过程中, 粒径 $D_{\mathrm{p}, j}$ 、密度 $\rho_{\mathrm{p}}$ 可表 示成剩余质量 $m_{\mathrm{cj}}$ 的函数 ${ }^{[19-20]}$

$$
\begin{aligned}
D_{\mathrm{p}, j} & =D_{\mathrm{p}, j}^{0}\left(m_{\mathrm{c} j} / m_{\mathrm{cj} j}^{0}\right)^{\alpha} \\
\rho_{\mathrm{p}} & =\rho_{\mathrm{p}}^{0}\left(m_{\mathrm{c} j} / m_{\mathrm{c} j}^{0}\right)^{\beta}
\end{aligned}
$$

式中, $3 \alpha+\beta=1$, 对于等密度燃烧模式 $\alpha=1 / 3$, $\beta=0$; 对于等直径燃烧模式 $\alpha=0, \beta=1$ 。

按式(16)解得 $m_{\mathrm{cj}}$ 后, 根据式(22)计算某一时刻 $t$ 的未燃烧碳总量 $C(\mathrm{~kg})$ 为

$$
C=\sum_{j=1}^{13} N_{j} m_{\mathrm{c} j}
$$

\section{4 氧气消耗方程}

炉膛内气流中的氧气被挥发分和煤焦燃烧所消 耗, 根据质量守恒方程, 气流氧气分压力 $P_{\mathrm{O}_{2}}$ 的变化 遵守如下方程 ${ }^{[20]}$

$$
\frac{1}{p_{0}} \frac{M_{\mathrm{O}_{2}}}{M_{\mathrm{g}}} m_{0} \frac{\mathrm{d} P_{\mathrm{O}_{2}}}{\mathrm{~d} t}=-\frac{1}{v_{\mathrm{v}}} \frac{\mathrm{d} V}{\mathrm{~d} t}-\frac{1}{v_{\mathrm{c}}} \sum_{j=1}^{13} N_{j} \frac{\mathrm{d} m_{\mathrm{c} j}}{\mathrm{~d} t}
$$

在煤粉进入炉膛的初始时刻, 满足

$$
t=0, \quad P_{\mathrm{O}_{2}}^{0}=0.2095 p_{0}
$$

式中, $p_{0}$ 为炉内燃烧压力, $p_{0}=101.325 \mathrm{kPa} ; M_{\mathrm{O}_{2}}$ 为 氧气摩尔质量, $M_{\mathrm{O}_{2}}=0.032 \mathrm{~kg} / \mathrm{mol} ; M_{\mathrm{g}}$ 为气体平均 摩尔质量, $\mathrm{kg} / \mathrm{mol} ; m_{0}$ 为对应 $1 \mathrm{~kg}$ 收到基煤, 截止 到时刻 $t$ 供给炉膛的空气质量, $\mathrm{kg}$; $v_{\mathrm{c}}$ 为当产物是 $\mathrm{CO}_{2}$ 时, $1 \mathrm{~kg}$ 氧气消耗掉碳的质量, $v_{\mathrm{c}}=0.375 \mathrm{~kg} ; v_{\mathrm{v}}$ 为 $1 \mathrm{~kg}$ 氧气消耗掉挥发分的质量, $\mathrm{kg}$, 见式(27); 
挥发分消耗速率 $\mathrm{d} V / \mathrm{d} t$ 见式(6); 煤焦碳颗粒消耗速 率 $\mathrm{d} m_{\mathrm{cj}} / \mathrm{d} t$ 见式(16)。

对式(23)进行离散化, 某一时刻烟气中氧气分 压力 $P_{\mathrm{O}_{2}}$ 可根据初始氧量压力 $P_{\mathrm{O}_{2}}^{0}$ 、挥发分和煤焦剩 余量 $V$ 和 $C$ 计算

$$
\frac{1}{p_{0}} \frac{M_{\mathrm{O}_{2}}}{M_{\mathrm{g}}} m_{0}\left(P_{\mathrm{O}_{2}}^{0}-P_{\mathrm{O}_{2}}\right)=\frac{1}{v_{\mathrm{v}}}\left(V_{\mathrm{f}}-V\right)+\frac{1}{v_{\mathrm{c}}}\left(C_{\mathrm{f}}-C\right)
$$

$1 \mathrm{~kg}$ 收到基煤在炉内热解产生的挥发分 $V_{\mathrm{f}} \mathrm{kg}$ 和 煤焦 $C_{\mathrm{f}} \mathrm{kg}, V_{\mathrm{f}} \mathrm{kg}$ 挥发分燃烧消耗的氧气记为 $m_{\mathrm{v}}$, $m_{\mathrm{v}}$ 等于 $1 \mathrm{~kg}$ 收到基煤完全燃烧消耗的氧气减去 $C_{\mathrm{f}} \mathrm{kg}$ 煤焦消耗的氧气, 根据燃烧化学反应计算可得到 $m_{\mathrm{v}}=8\left(C_{\mathrm{ar}} / 100-C_{\mathrm{f}}\right) / 3+\left(S_{\mathrm{ar}}+8 H_{\mathrm{ar}}-O_{\mathrm{ar}}\right) / 100$

因此, 式(24)、(25)中 $1 \mathrm{~kg}$ 氧气消耗掉挥发分 的质量 $v_{\mathrm{v}}$ 为

$$
v_{\mathrm{v}}=V_{\mathrm{f}} / m_{\mathrm{v}}
$$

\section{5 煤粉颗粒停炉时间}

假设烟气在炉内为柱塞流动, 煤粉颗粒在炉膛 某个区段 $i$ 中的停留时间为

$$
t_{i}=\frac{V_{i} T_{0}}{T_{\mathrm{g}} Q_{i} B}
$$

式中, $t_{\mathrm{i}}$ 为颗粒在区段 $i$ 中的停留时间, $\mathrm{s} ; V_{\mathrm{i}}$ 为区 段 $i$ 的体积, $\mathrm{m}^{3} ; T_{0}$ 为标准状态温度, $T_{0}=293.15 \mathrm{~K}$; $T_{\mathrm{g}}$ 为区段 $i$ 中的烟气平均温度, $\mathrm{K} ; Q_{\mathrm{i}}$ 为在区段 $i$ 中过剩空气系数和煤燃尽度下, $1 \mathrm{~kg}$ 煤产生的烟气 在标准状态的体积, $\mathrm{Nm}^{3} / \mathrm{kg} ; B$ 为锅炉燃料消耗量, $\mathrm{kg} / \mathrm{s}$ 。

根据柱塞流动假设, 还可以将燃烧时间 $t$ 换算 成炉膛垂直方向距离 $h$ 。

\section{6 燃烧方程求解}

根据煤粉细度 $R_{90}$ 和 $R_{200}$ 的值, 利用式(2)、(3)、 (12)计算 13 个颗粒组分的质量份额; 根据煤的成分, 利用式(4)、(9)、(10)计算热解参数, 然后, 在某个 炉膛区段 $i$ 上, 求解煤粉燃烧方程即式(6)、(16), 求解时, 颗粒温度 $T_{\mathrm{p}}$ 和气体温度 $T_{\mathrm{g}}$ 作为已知参数, 它们从炉膛分区段传热计算中获得; 从煤粉进入炉 膛的时刻 $t=0$ 开始, 根据挥发分和煤焦的初始值, 计算经过时间间隔 $\Delta t$ 的质量变化 $\Delta V$ 和 $\Delta m_{\mathrm{cj}}$; 对于煤 焦颗粒, 对 13 种粒径组分分别进行计算, 得到每种 粒径的剩余量 $m_{\mathrm{cj}}$, 并根据式(22)对所有粒径组分进 行累积, 得到未燃尽的煤焦碳总量 $C$ 以及未燃尽挥 发分量 $V$, 在该时间间隔的末尾, 利用式(25)得到烟 气中氧气分压力 $P_{\mathrm{O}_{2}}$, 重新开始下一时间间隔的计 算, 直到该区段的停留时间 $t_{\mathrm{i}}$ 结束, 得到所有时刻
未燃尽挥发分质量 $V$ 和煤焦碳质量 $m_{\mathrm{cj}} 、 C$ 。

\section{7 颗粒燃烬指标计算}

根据方程(6)和式(16)解得的挥发分剩余量 $V$ 和 煤焦剩余碳量 $m_{\mathrm{cj}}$, 可以计算颗粒的各种燃烬指标, 其中一些指标也通过试验进行测定。

（1）煤粉未燃烬度 $\chi$ 。煤粉未燃尽度 $\chi$ 定义为 未燃烬的可燃物质量与原有可燃物（包括挥发分和 煤焦)质量的比

$$
\chi=\frac{C+V}{C_{\mathrm{f}}+V_{\mathrm{f}}}
$$

（2）煤焦颗粒未燃尽度。对于某个粒径 $j$, 煤焦 颗粒未燃烬度定义为颗粒中剩余碳质量 $m_{\mathrm{cj}}$ 与初始 碳质量 $m_{\mathrm{cj}}^{0}$ 之比

$$
u_{j}=m_{c j} / m_{\mathrm{cj}}^{0}
$$

煤焦颗粒群整体未燃烬度定义为颗粒群中剩余 碳量与初始碳量之比

$$
U=\sum_{j=1}^{13} N_{j} m_{\mathrm{cj}} / C_{\mathrm{f}}
$$

（3）飞灰可燃物含量 $C_{\mathrm{fh}}$ 。在实际生产中, 通常 采用飞灰中可燃物质量占飞灰质量的百分数表示颗 粒燃烬程度, 称为飞灰可燃物含量 $C_{\mathrm{fh}}$, 它与煤粉未 燃烬度 $\chi$ 的关系为

$$
C_{\mathrm{fh}}=\frac{100 \chi\left(C_{\mathrm{f}}+V_{\mathrm{f}}\right)}{A_{\mathrm{ar}}+\chi\left(C_{\mathrm{f}}+V_{\mathrm{f}}\right)}
$$

忽略飞灰中未燃烬的挥发分, 则飞灰可燃物含 量 $C_{\mathrm{fh}}$ 与煤焦颗粒群未燃烬度 $U$ 的关系为

$$
C_{\mathrm{fh}}=100 U C_{\mathrm{f}} /\left(U C_{\mathrm{f}}+0.01 A_{\mathrm{ar}}\right)
$$

(4) 燃料热量释放率。燃料热量释放率 $\beta$ 定义 为燃料释放出的热量与燃料中原有热量之比, 按下 式计算

$$
\beta=1-\left(q_{\mathrm{c}} C+q_{\mathrm{v}} V\right) / Q_{\mathrm{ar}, \mathrm{net}, \mathrm{p}}
$$

式中, $Q_{\mathrm{ar}, \mathrm{net}, \mathrm{p}}$ 为煤收到基低位发热量, $\mathrm{kJ} / \mathrm{kg} ; q_{\mathrm{c}}$ 为 $1 \mathrm{~kg}$ 碳燃烧放出的热量, $q_{\mathrm{c}}=33727 \mathrm{~kJ} / \mathrm{kg} ; q_{\mathrm{v}}$ 为 $1 \mathrm{~kg}$ 挥发分燃烧放出的热量, $\mathrm{kJ} / \mathrm{kg}$, 见式(35)。

$1 \mathrm{~kg}$ 收到基煤在炉膛释放出的挥发分为 $V_{\mathrm{f}} \mathrm{kg}$, 这些挥发分的发热量等于原煤收到基发热量 $Q_{\mathrm{ar}, \mathrm{net}, \mathrm{p}}$ 与 $C_{\mathrm{f}} \mathrm{kg}$ 煤焦发热量之差, 因此 $1 \mathrm{~kg}$ 挥发分 燃烧放出的热量 $q_{\mathrm{v}}$ 为

$$
q_{\mathrm{v}}=\left(Q_{\mathrm{ar}, \text { net }, \mathrm{p}}-33727 C_{\mathrm{f}}\right) / V_{\mathrm{f}}
$$

（5）机械不完全燃烧损失 $q_{4}$ 。机械不完全燃烧 损失 $q_{4}$ 是煤粉中可燃物未完全燃烧损失热量的百 分数, 热量释放率 $\beta$ 与 $q_{4}$ 的关系为 


$$
q_{4}=100(1-\beta)
$$

根据式(34)得到热量释放率 $\beta$ 后，代入式(36) 计算 $q_{4}$, 然后利用 $q_{4}$ 根据下式可计算飞灰可燃物含 量 $C_{\mathrm{fh}}$

$$
C_{\mathrm{fh}}=100 q_{4} /\left(q_{4}+33727 A_{\mathrm{ar}} / Q_{\mathrm{ar}, \mathrm{net}, \mathrm{p}}\right)
$$

\section{2 改进的炉膛分区段传热计算}

煤粉燃烧方程中烟气和颗粒温度, 采用炉膛分 区段传热模型确定。

分区段传热计算时, 炉膛被分为若干个区段, 在每个区段上, 采用先假定后校正的方法, 求解包 含辐射换热、煤热量释放以及烟气进出口焓值变化 的热平衡方程, 得到区段出口烟气温度 ${ }^{[5]}$; 传统的 分区段传热计算适用于常规非空气分级燃烧, 这种 燃烧方式在主燃烧区一次性供给所有的燃烧空气, 煤在主燃烧区的热量释放率达 $95 \%$ 以上，各区段的 热量释放率根据煤质选用经验数据, 飞灰浓度、三 原子气体含量等烟气辐射成分含量则是按照煤完全 燃烧考虑。

对于空气分级燃烧, 主燃烧区煤热量释放率受 该区供给的空气量控制, 对于烟煤, 该热量释放率 仅在 70\%左右, 因此分区段传热计算不适用于空气 分级燃烧, 为此, 对传统分区段传热模型进行如下 修订: 在各区段上, 煤热量释放率通过求解燃烧模 型获得, 烟气辐射成分参数根据煤的实际燃烬程度 计算; 修订后热平衡方程为

$$
\begin{gathered}
\vartheta^{\prime \prime} V_{\mathrm{cp}}^{\prime \prime}=\beta_{\mathrm{m}} Q_{\mathrm{ar}, \mathrm{net}, \mathrm{p}}+Q_{\mathrm{ap}}-Q_{6}-Q_{\mathrm{rm}} \\
\vartheta^{\prime \prime} V_{\mathrm{c}}^{\prime \prime}-V_{\mathrm{c}}^{\prime} \vartheta^{\prime}=\left(\beta^{\prime \prime}-\beta^{\prime}\right) Q_{\mathrm{ar}, \text { net }, \mathrm{p}}+Q_{\mathrm{a}}-Q_{\mathrm{r}} \\
Q_{\mathrm{rm}}=M \sigma_{0} a_{\mathrm{t}} T^{\prime \prime 4} \psi F / B \\
Q_{\mathrm{r}}=M\left[1+\left(\frac{T^{\prime \prime}}{T^{\prime}}\right)^{4}\right] \frac{\sigma_{0} a_{\mathrm{t}} T^{\prime 4}}{2 B}\left(F_{\mathrm{pj}} \Delta \psi+\psi_{\mathrm{cp}} F_{\mathrm{w}}\right)
\end{gathered}
$$

式(38)适用于主燃烧区，对于主燃烧区以外的 其他燃烧区段, 则采用式(39)。式中， $\vartheta^{\prime} 、 T^{\prime}$ 为区 段入口烟气的摄氏温度和绝对温度, $\vartheta^{\prime}+273=1 T 5 ; \vartheta^{\prime \prime} 、 T^{\prime \prime}$ 为区段出口烟气的摄氏温 度和绝对温度, $\vartheta^{\prime \prime}+273.15=T^{\prime \prime} ; V_{\mathrm{cp}}^{\prime \prime} 、 V_{\mathrm{c}}^{\prime \prime}$ 为在区段 出口实际燃尽度和烟气温度下, 每千克入炉煤产生 烟气的平均热容量, $\mathrm{kJ} /\left(\mathrm{kg} \cdot{ }^{\circ} \mathrm{C}\right) ; V_{\mathrm{c}}^{\prime}$ 为在区段入口 实际燃尽度和烟气温度下, 每千克入炉煤产生烟气 的平均热容量, $\mathrm{kJ} /\left(\mathrm{kg} \cdot{ }^{\circ} \mathrm{C}\right) ; \beta_{\mathrm{m}}$ 为煤在主燃烧区的 燃烬度; $\beta^{\prime} 、 \beta^{\prime \prime}$ 为分别为区段进出、口煤的燃尽 度; $Q_{\mathrm{ap}}$ 为进入区段的空气带入的热量, $\mathrm{kJ} / \mathrm{kg} ; Q_{6}$ 为 灰渣物理热损失, $\mathrm{kJ} / \mathrm{kg} ; Q_{\mathrm{a}}$ 为进入区段的空气带入
的热量, $\mathrm{kJ} / \mathrm{kg} ; Q_{\mathrm{r}}$ 为区段的辐射换热流量, $\mathrm{kJ} / \mathrm{kg}$; $Q_{\mathrm{rm}}$ 为主燃烧区的辐射换热流量, $\mathrm{kJ} / \mathrm{kg} ; M$ 为温度 场不均的修正系数; $\sigma_{0}$ 为黑体辐射常数, $\sigma_{0}=5.67 \times 10^{-11} \mathrm{~kJ} /\left(\mathrm{m}^{2} \cdot \mathrm{s} \cdot \mathrm{K}^{4}\right) ; a_{\mathrm{t}}$ 为该区段炉膛的 黑度, 采用实际燃烬度计算; $F_{\mathrm{pj}}$ 为该区段炉膛的平 均横截面积, $\mathrm{m}^{2} ; F_{\mathrm{W}}$ 为该区段炉墙总面积, $\mathrm{m}^{2} ; \psi$ 为热有效系数; $\Delta \psi$ 为该区段进出口辐射热系数之 差, $\Delta \psi=\psi^{\prime \prime}-\psi^{\prime} ; \psi_{\mathrm{cp}}$ 为该区段炉壁的平均热有效 系数。

改进后的炉膛分区段传热计算模型不仅适用于 空气分级燃烧, 还适用于非空气分级燃烧, 对于空 气分级燃烧，式(23)、(25)中的 $m_{0}$ 在主燃烧区和燃 烬区是不同的，而对于非空气分级燃烧， $m_{0}$ 在主燃 烧区以上保持不变, 因为该燃烧方式的全部空气从 主燃烧区供入。

分区段传热计算模型经过长期工程应用检验, 烟气温度计算精度在 $\pm 3 \%$ 范围 ${ }^{[21]}$, 但随着锅炉容量 增加, 炉膛零维和分区段传热模型总是过高估计了 换热量, 主要原因是, 随着炉膛尺寸增加, 温度场 不均匀性增大; 因此, 与传统分区段传热计算相比, 式(40)、(41)的另一点不同是, 采用修正系数 $M$ 考 虑温度场不均匀性引起辐射换热的降低 ${ }^{[22]}$

$$
M=6068 /\left(T^{\prime \prime} T_{\mathrm{a}}^{0.2}\right)
$$

式中, $T_{\mathrm{a}}$ 为煤的绝热燃烧温度, $\mathrm{K}$ 。

\section{3 燃烧和传热耦合求解}

燃烧和传热方程的耦合求解步骤如下: 求解时, 先从主燃烧区开始，对于每个区段，假定区段出口 的烟气温度和煤热量释放率, 根据式(40)或式(41) 计算辐射换热量, 利用式(38)或式(39)计算区段出口 烟气温度, 根据区段进、出口烟气温度, 求解煤粉 燃烧方程(6)、(16), 得到该区段煤热量释放率, 当 热量释放率的假设值和计算值偏差小于 0.0005 , 且 出口烟气温度的假设值和计算值偏差小于 $0.1 \mathrm{~K}$, 该 区段计算结束; 当所有区段的计算偏差满足上述要 求后, 整个炉膛计算结束。

求解煤粉燃烧方程时, 认为煤粉在区段中等温 燃烧, 在主燃烧区, 当挥发分析出 $60 \%$ 以上时, 煤 焦颗粒的燃烧反应开始进行，据此可确定式(19)中 的时刻 $t_{\mathrm{c}}$; 在热解计算时, 式(4)、(7)中的颗粒温 度 $T_{\mathrm{p}}$ 等于主燃烧区进口烟气温度 $T^{\prime}$, 在煤焦颗粒燃 烧计算时, 式(17)、(18)中的颗粒温度 $T_{\mathrm{p}}$, 以及式(18)、 (28)中的气体温度 $T_{\mathrm{g}}$, 均等于区段进、出口烟气温 度 $T^{\prime} 、 T^{\prime \prime}$ 的平均值; 式(23)、(25)中气体平均摩尔 
质量 $M_{\mathrm{g}}$ 根据煤在实际燃烬度下的烟气成分计算; 根据式(6)和(16)解得颗粒中剩余可燃物质量 $V$ 和 $m_{\mathrm{cj}}$ 后, 利用式(29) (37)进行各种燃烬指标计算, 以便于分析和对比。

\section{4 实炉试验及模拟计算}

\section{1 设备概况}

试验对象为一台空气分级低 $\mathrm{NO}_{x}$ 切向燃烧烟煤 锅炉, 型号为 SG-1025.7/18.3-M840, 是亚临界压力、 中间一次再热、控制循环汽包锅炉, 采用正压直吹 式制粉系统, 配有 5 台 RP923 磨煤机, 燃烧器采用 四角切向布置, 如图 1, 煤粉喷嘴共 5 层, 从下至 上依次记为 $A 、 B 、 C 、 D 、 E$ 层, 空气喷嘴包括: (1) 位于最底层的 $\mathrm{AA}$ 二次风, (2) $\mathrm{CD}, \mathrm{DE}$ 和 $\mathrm{EE}$ 等 3 层辅助风, (3) $\mathrm{AB}, \mathrm{BC}$ 等 2 层油枪二次风, (4) $\mathrm{A}$ 、 B、C、D、E 等 5 层周界风，(5) SOFA1、SOFA2、 SOFA3 和 SOFA4 等 4 层分离燃尽风, 炉膛分为主燃 烧区和分离燃尽风区, 进入炉膛的总空气量为 $\alpha_{\mathrm{f}}$, 其中从主燃烧区送入的空气量为 $\alpha_{\mathrm{p}}$, 其余的空气从 SOFA 燃尽区送入, 形成分级燃烧, 主燃烧区最上层 空气喷嘴和最底层分离燃尽风喷嘴中心线之间的垂 直距离为 $6 \mathrm{~m}$; 图 1 是炉膛结构和燃烧器布置。
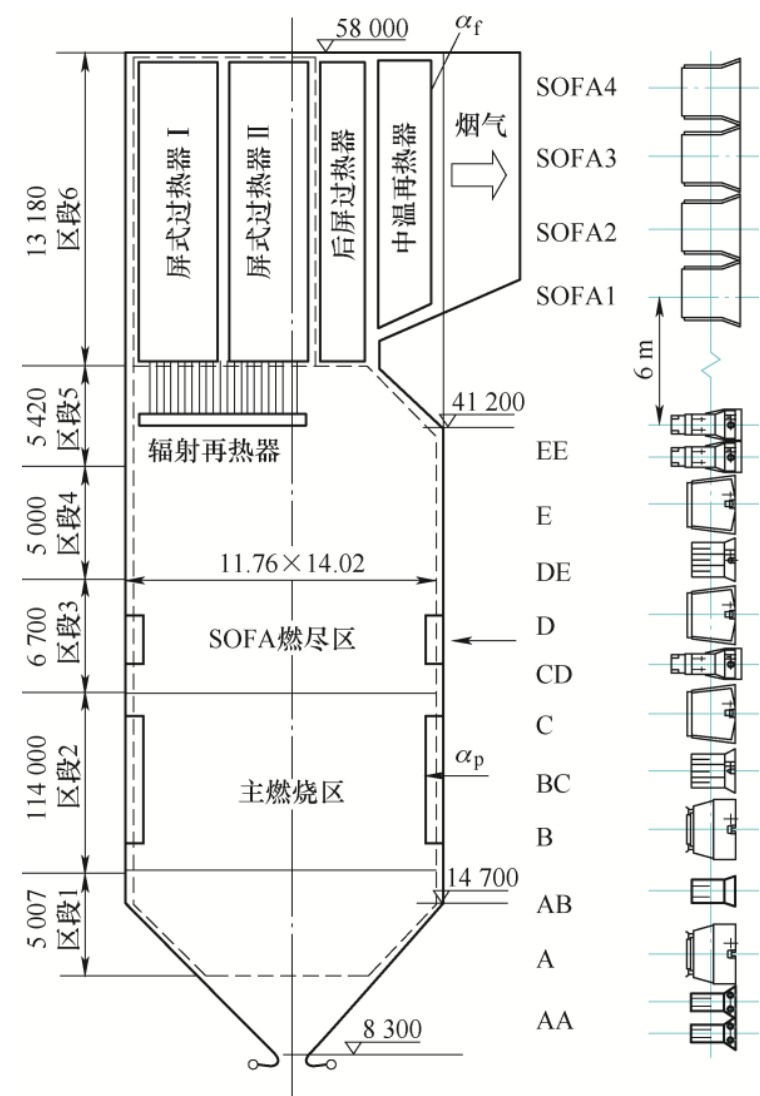

图 1 炉膛及燃烧器布置
在炉膛分区段传热计算时，将炉膛在垂直方向 上分为图 1 所示的 6 个区域, 其中区段 6 靠近炉膛 顶部且布置的受热面较多, 烟气温度明显降低, 该 区不进行燃烧计算, 认为区段 5 出口就是炉膛出口 的颗粒燃烬水平。

\section{2 试验数据}

实炉试验目的是测定燃烧和传热模型的输入参 数, 并在炉膛出口采集飞灰样品, 化验可燃物含量, 以便于与模型预测结果进行对比。

试验在锅炉全负荷运行范围内进行 ${ }^{[23]}$, 按照 GB/T 10184-2015 标准 ${ }^{[24]}$ 规定, 进行煤、煤粉和飞 灰的采样化验分析, 采用 NOVA2000 烟气分析仪测 量炉膛出口烟气含氧量, 用于计算过剩空气系数, 主蒸汽流量、燃煤量和进风温度等参数利用机组控 制系统记录。

为确定主燃烧区和分离燃尽风区进入炉膛的 二次风流量, 利用文献[25]中以二次风喷口挡板 作为测量元件的风量测量系统, 但试验前需对挡 板阻力系数进行标定; 从煤粉喷嘴进入主燃烧区 的一次风通过测量磨煤机的通风流量确定, 该流 量在磨煤机入口利用经过标定的靠背管测量; 从 主燃烧区进入的总空气流量等于一、二次风流量 之和。

试验过程中, 煤质保持稳定, 表 1 给出煤和煤 粉细度采样化验结果, 表 2 给出不同负荷下主要试 验数据。

\section{表 1 煤和煤粉分析}

\begin{tabular}{|c|c|c|}
\hline & 项目 & 数据 \\
\hline \multirow{8}{*}{$\begin{array}{l}\text { 元素分析 } \\
\text { (收到基) }\end{array}$} & 碳(\%) & 55.54 \\
\hline & 氢(\%) & 3.14 \\
\hline & 氧(\%) & 3.95 \\
\hline & 氮(\%) & 1.15 \\
\hline & 硫(\%) & 1.8 \\
\hline & 灰分 $(\%)$ & 26.92 \\
\hline & 水分 $(\%)$ & 7.6 \\
\hline & 收到基低位发热量 $/\left(\mathrm{kJ} \cdot \mathrm{kg}^{-1}\right)$ & 19629 \\
\hline \multirow{4}{*}{$\begin{array}{l}\text { 工业分析 } \\
\text { (空气干 } \\
\text { 燥基) }\end{array}$} & 固定碳(\%) & 46.06 \\
\hline & 挥发分(\%) & 23.04 \\
\hline & 灰分 $(\%)$ & 28.41 \\
\hline & 水分 $(\%)$ & 2.50 \\
\hline \multirow{2}{*}{ 煤粉细度 } & R90(\%) & 26.0 \\
\hline & R200(\%) & 3.0 \\
\hline
\end{tabular}


表 2 主要试验数据

\begin{tabular}{|c|c|c|c|c|c|}
\hline 项目 & 工况 1 & 工况 2 & 工况 3 & 工况 4 & 工况 5 \\
\hline 机组负荷/ MWe & 300.8 & 270.2 & 240.3 & 210.0 & 180.6 \\
\hline 主蒸汽流量/ $\left(\mathrm{t} \cdot \mathrm{h}^{-1}\right)$ & 911.6 & 808.2 & 710.8 & 612.1 & 496.9 \\
\hline 一次风流量 $/\left(\mathrm{t} \cdot \mathrm{h}^{-1}\right)$ & 304.0 & 304.5 & 319.7 & 311.9 & 212.7 \\
\hline 一次风温度 $/{ }^{\circ} \mathrm{C}$ & 65 & 71 & 70 & 69 & 68 \\
\hline 主燃烧区空气流量/(t・ $\left.\mathrm{h}^{-1}\right)$ & 637.0 & 594.0 & 613.9 & 540.2 & 404.1 \\
\hline SOFA 风区空气流量 $/\left(\mathrm{t} \cdot \mathrm{h}^{-1}\right)$ & 410.3 & 294.8 & 284.0 & 239.8 & 237.1 \\
\hline 二次风温度/ ${ }^{\circ} \mathrm{C}$ & 303.9 & 303.6 & 295.1 & 291.4 & 286.1 \\
\hline 锅炉燃煤量 $/\left(\mathrm{t} \cdot \mathrm{h}^{-1}\right)$ & 124.2 & 108.1 & 100.3 & 84.8 & 65.5 \\
\hline 炉膛出口过剩空气系数 & 1.17 & 1.14 & 1.23 & 1.26 & 1.33 \\
\hline 主燃烧区过剩空气系数 & 0.71 & 0.76 & 0.84 & 0.87 & 0.84 \\
\hline 主燃烧区空气量 $m_{0 \mathrm{~m}} / \mathrm{kg}$ & 8.48 & 8.36 & 9.04 & 9.29 & 9.89 \\
\hline 分离燃尽风区空气量 $m_{0 \mathrm{~s}} / \mathrm{kg}$ & 5.16 & 5.59 & 6.18 & 6.43 & 6.23 \\
\hline $\mathrm{A}$ 侧飞灰含碳量(\%) & 1.36 & 3.07 & 2.42 & 2.23 & 0.72 \\
\hline $\mathrm{B}$ 侧飞灰含碳量 $(\%)$ & 0.65 & 3.92 & 1.38 & 1.83 & 2.4 \\
\hline
\end{tabular}

根据主燃烧区和分离燃尽风区的空气流量, 可 得到氧量方程式(23)中的单位入炉煤所对应的炉膛 空气量 $m_{0}$, 结果见表 2 。

\section{3 模型其他参数设定}

除表 $1 、 2$ 给出的试验数据外, 燃烧和传热模型 中其他参数的设定如下。

式(13)中的膨胀因子 $\delta=1$ 。

式(14)中的煤焦颗粒的初始密度 $\rho_{\mathrm{p}}^{0}=887 \mathrm{~kg} / \mathrm{m}^{3}$ 。

式(20)中的燃烧模式为等密度模式 $\alpha=1 / 3$, 或等 直径模式 $\alpha=0$ 。

式(18)中计算扩散反应速率系数时的反应机理 因子 $\varphi=2$ 。

煤焦颗粒表面反应频率因子 $A_{\mathrm{f}}=0.004974$ $\mathrm{kg} /\left(\mathrm{m}^{2} \cdot \mathrm{s} \cdot \mathrm{Pa}\right)$, 反应活化能为 $E_{\mathrm{a}}=74092.2 \mathrm{~J} / \mathrm{mol}$, 本文采用的煤种挥发分与文献[26]中的 Whitwick 煤 接近, $A_{\mathrm{f}}$ 和 $E_{\mathrm{a}}$ 是该煤种的数据。

表 $1 、 2$ 中现场测定的锅炉运行数据作为燃烧传 热模型的输入参数, 采用该模型对炉膛出口颗粒然 烬度进行数值模拟, 计算是在 Excel VBA 平台上进 行, 简单运算通过 Excel 单元格之间的相互调用完 成, 空气和烟气性质以及煤理论燃烧温度等参数采 用自创 Excel 函数计算, 煤粉燃烧模型则在独立模 块中运算, 通过 BeforeDoubleClick 工作表双击事件 启动计算程序, 当各区段出口烟气温度和颗粒燃烬 率的计算偏差小于给定微小量, 计算结束。

除进行表 2 中 5 个模拟工况外, 还假设表中 $300.8 \mathrm{MW}$ 负荷下所有空气均从主然烧区送入, 即进 行了该负荷下的非空气分级工况计算。

\section{5 分析和讨论}

\section{1 空气分级燃烧和非空气分级燃烧}

对于 $300.8 \mathrm{MW}$ 负荷下的空气分级燃烧和非空 气分级燃烧, 图 2 给出煤热量释放率和烟气含氧量 沿炉膛高度的变化, 可以看出, 非空气分级燃烧时, 主燃烧区消耗掉大部分的氧气，主燃烧区出口煤热 量释放率接近 0.98 , 烟气含氧量和煤热量释放率 $\beta$ 沿炉膛高度连续变化, 炉膛出口处煤热量释放率为 0.996; 而对于空气分级燃烧，因主燃烧区过剩空气 系数为 0.71 , 主燃烧区很快消耗掉烟气中所有氧气, 在 $h=9.5 \sim 16.41 \mathrm{~m}$ 范围内, 烟气含氧量为 0 , 煤热 量释放率保持在 0.66 不变, 直到 SOFA 风区补充新 鲜空气后, 燃烧才进一步进行, 炉膛出口处煤热量 释放率达到 0.990 。

对于非空气分级燃烧, 文献[5]给出煤热量释放 率沿炉膛高度的推荐值, 见图 2, 从图中可看出, 推荐值和模型预测值吻合较好。

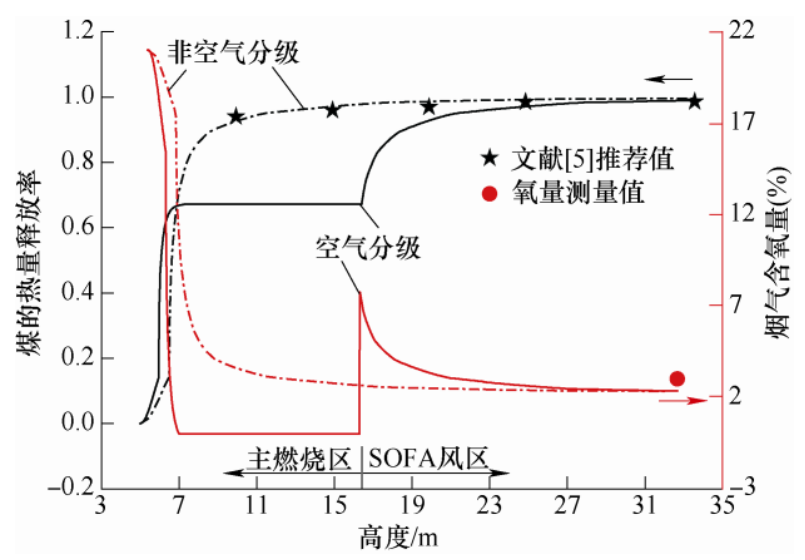

图 2 煤热量释放率和烟气含氧量沿炉膛高度的变化

对于空气分级燃烧工况，图 2 还给出炉膛出口 烟气含氧量的测量值, 氧气分析仪从炉内抽吸烟气 进行采样, 测得的氧气含量接近干烟气含氧量, 因 此, 其数值略大于模拟计算得到的包含水蒸气在内 的烟气含氧量。

图 3 给出表 2 中 5 个工况以及 $300.8 \mathrm{MW}$ 负荷 的非空气分级燃烧工况下, 烟气温度随炉膛高度变 化。可以看出, 由于 $300.8 \mathrm{MW}$ 负荷非空气分级燃 烧工况下主燃烧区煤热量释放率较高, 因此, 主燃 烧区出口烟气温度比相同负荷下的空气分级工况高 $78{ }^{\circ} \mathrm{C}$; 对于表 2 中 5 个空气分级燃烧工况，主然烧 区出口烟气温度不仅决定于锅炉负荷，还受主燃烧 区供给空气量的影响，因为煤热量释放率由该空气 量决定，从图 3 中可看出, $240.3 \mathrm{MW}$ 负荷下主燃烧 
区出口烟气温度为 $1438{ }^{\circ} \mathrm{C}$, 高于 $300.8 \mathrm{MW}$ 负荷 下的 $1423{ }^{\circ} \mathrm{C}$, 主要原因是 $240.3 \mathrm{MW}$ 负荷下主燃烧 区过剩空系数为 0.84 , 而 $300.8 \mathrm{MW}$ 负荷时为 0.71 , 前者主燃烧区煤热量释放率较高。

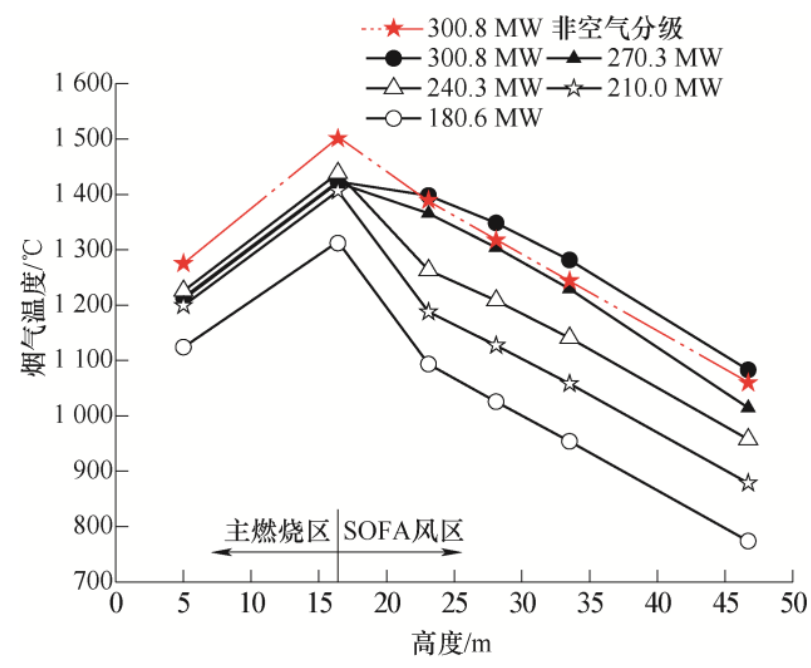

图 3 不同工况下烟气温度沿炉膛高度的变化

图 4、5 分别给出空气分级燃烧和非空气分级燃 烧工况下, 不同煤焦颗粒燃尽度沿炉膛高度的变化, 可以看出, 对于空气分级燃烧, 粒径在 $33 \mu \mathrm{m}$ 以下 的颗粒在主燃烧区完全燃烬, 在 SOFA 风区补充供 给空气后, 粒径 $168 \mu \mathrm{m}$ 以下的颗粒在炉膛出口完 全燃烬, 而粒径 $213 \mu \mathrm{m}$ 以上颗粒在炉膛出口不能 燃烬, 这些颗粒是飞灰中碳的主要来源, 炉膛出口 的煤焦颗粒总燃尽度为 0.987 , 飞灰中可燃物含量为 2.10\%; 对于非空气分级燃烧, 粒径 $168 \mu \mathrm{m}$ 以下的 颗粒在主燃烧区完全燃烬, 在炉膛出口处, 只有粒 径为 $255 \mu \mathrm{m}$ 和 $350 \mu \mathrm{m}$ 两种粒径的颗粒未能燃烬, 炉膛出口处煤焦颗粒总燃尽度为 0.995 , 飞灰中可燃 物含量为 $0.77 \%$ 。

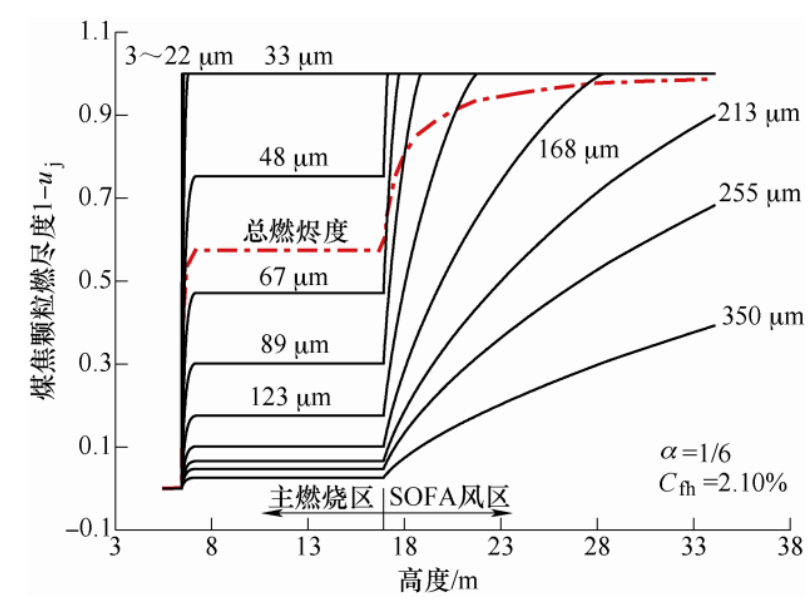

图 $4300.3 \mathrm{MW}$ 负荷空气分级工况下不同颗粒的燃烬度

根据燃烧模拟计算结果, 以表 2 中工况 1 为例, 空气分级炉内煤粉燃烬过程如下: 煤粉在炉膛内总
计停留 $4.1 \mathrm{~s}$, 其中在主燃烧区停留 $2.2 \mathrm{~s}$, 全部挥发 分的析出和燃烧在 $0.4 \mathrm{~s}$ 内完成, 粒径 $33 \mu \mathrm{m}$ 以下的 煤粉颗粒在 $0.35 \mathrm{~s}$ 内全部燃尽，在主燃烧区后部 $1.8 \mathrm{~s}$, 烟气中氧气被全部消耗, 所有煤粉颗粒均处 于燃烧停滞状态, 主燃烧区出口煤粉燃烬度为 0.69 , 热量释放率为 0.65 ; 在分离燃烬风区补充新鲜空气 后, 粒径 $123 \mu \mathrm{m}$ 以下的颗粒在 $0.53 \mathrm{~s}$ 内全部燃烬, $213 \mu \mathrm{m} 、 255 \mu \mathrm{m}$ 和 $350 \mu \mathrm{m}$ 等三种颗粒在炉膛出口 未能燃烬, 颗粒组分的煤焦燃烬度分别为 $0.90 、 0.68$ 和 0.39 , 由于它们所占质量份额仅为 0.057 , 炉膛出 口处煤粉总体燃烬度和热量释放率仍较高, 均达到 0.99 。

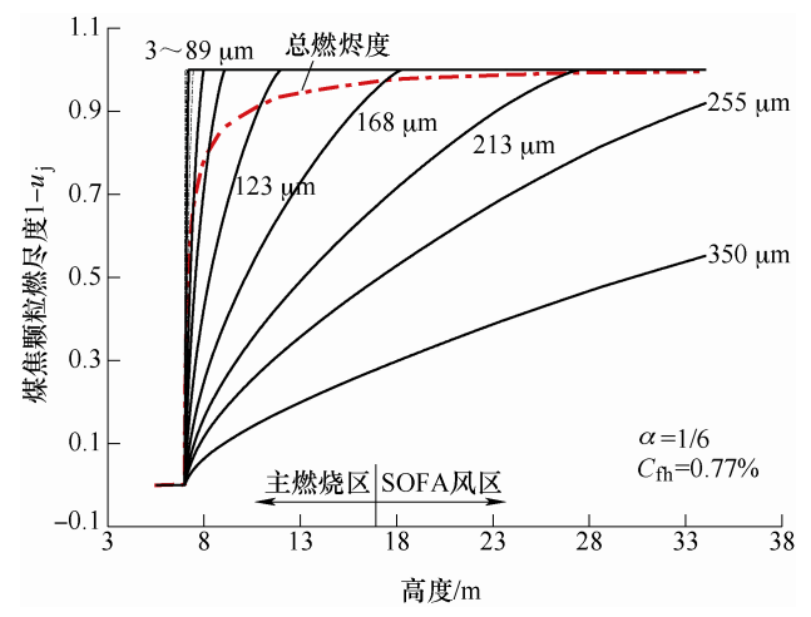

图 $5300.3 \mathrm{MW}$ 负荷非空气分级工况下不同颗粒的燃烬度

\section{$5.2 飞$ 灰可燃物含量预测值和实测值对比}

图 6 给出了表 2 中不同负荷下，飞灰可燃物含 量的预测值和实际采样化验值的对比, 预测计算在 等密度 $\alpha=1 / 3$ 和等直径 $\alpha=0$ 两种燃烧模式下进行, 可以看出, 等直径模式的预测值总是小于等密度模 式，这是因为在等密度模式下，煤焦颗粒燃烧过程 中收缩, 燃烧比表面积减少; 实际采样化验的飞灰 可燃物含量与等密度燃烧模式的预测结果更为接

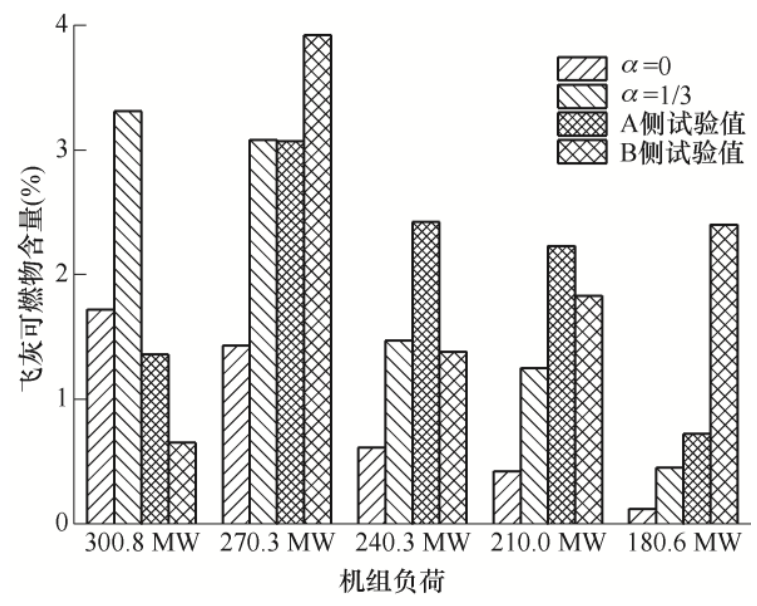

图 6 不同负荷下飞灰可燃物的预测值和测量值 
近, 在 $300.8 \mathrm{MW} 、 270.3 \mathrm{MW} 、 240.3 \mathrm{MW}$ 和 $210.0 \mathrm{MW}$ 四个负荷下, 预测值与化验结果吻合较 好, 在 180.6 MW 工况下, 预测值低于化验值较多。

\section{3 飞灰可燃物含量预测值的影响因素分析}

飞灰可燃物含量预测值除与燃烧模式 $\alpha$ 有关 外, 还与煤焦表面反应速率参数即方程式(17)中的 $E_{\mathrm{a}}$ 和 $A_{\mathrm{f}}$ 有关, 目前, 不同文献中给出的 $E_{\mathrm{a}} 、 A_{\mathrm{f}}$ 值差 别较大, 表 3 是一部分文献中给出烟煤的 $E_{\mathrm{a}}$ 和 $A_{\mathrm{f}}$ 值, 表中还给出两个燃烧温度下 $\left(1000{ }^{\circ} \mathrm{C}\right.$ 和 $\left.1300{ }^{\circ} \mathrm{C}\right)$ 计算所得的表面化学反应速率系数 $R_{\mathrm{c}}$, 可以看出, 在相同温度下, 表面化学反应速率系数差别较大。

表 3 不同文献给出的煤焦表面反应速度参数

\begin{tabular}{ccccccc}
\hline $\begin{array}{c}\text { 代 } \\
\text { 号 }\end{array}$ & 文献 & $\begin{array}{c}E_{\mathrm{a}} l \\
(\mathrm{~J} / \mathrm{mol})\end{array}$ & $\begin{array}{c}A_{\mathrm{f}} / \\
\left(\mathrm{kg} / \mathrm{m}^{2} \cdot \mathrm{s} \cdot \mathrm{Pa}\right)\end{array}$ & $\begin{array}{c}R_{\mathrm{c}}\left(1000{ }^{\circ} \mathrm{C}\right) \\
/ \times 10^{-7}\end{array}$ & $\begin{array}{c}R_{\mathrm{c}}\left(1300{ }^{\circ} \mathrm{C}\right) \\
/ \times 10^{-7}\end{array}$ & $\begin{array}{c}\text { 煤种或挥 } \\
\text { 发分 }(\%)\end{array}$ \\
\hline I & {$[26]$} & 100883 & 0.010984 & 7.964021 & 49.04149 & $V_{\mathrm{daf}}=36.4$ \\
II & {$[26]$} & 89999 & 0.006938 & 14.0667 & 71.19636 & $\begin{array}{c}V_{\mathrm{daf}}=37.9 \\
\text { 除无烟煤 }\end{array}$ \\
III & {$[10]$} & 149440 & 0.859800 & 6.342094 & 93.67801 & $\begin{array}{c}\text { 之外的煤 } \\
\text { 种 }\end{array}$ \\
IV & {$[26]$} & 74092 & 0.004974 & 45.33072 & 172.2592 & $V_{\mathrm{daf}}=37$ \\
V & {$[27]$} & 69007 & 0.005777 & 85.1138 & 295.121 & $V_{\mathrm{daf}}=34.4$ \\
\hline
\end{tabular}

以表 2 中工况 1 的测试数据作为模型输入数据, 在不同的燃烧模式 $\alpha$ 下, 利用表 3 中的 $E_{\mathrm{a}} 、 A_{\mathrm{f}}$ 值对 颗粒燃烬状态进行模拟计算，得到的结果见图 7 。

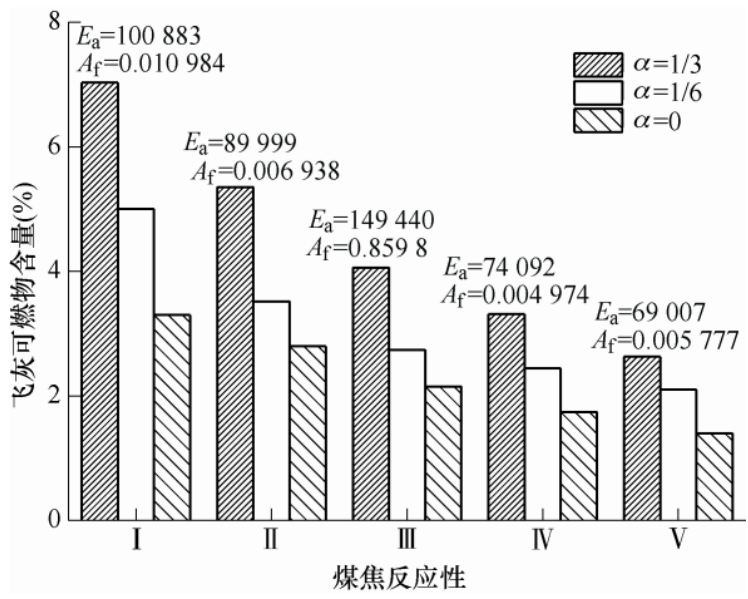

图 7 不同燃烧模式 $\alpha$ 及 $E_{\mathrm{a}} 、 A_{\mathrm{f}}$ 参数下的飞灰可燃物含量

从图 7 可看出, $\alpha=1 / 6$ 时, 飞灰可燃物含量预 测值介于等密度和等直径模式之间, 在相同的 $E_{\mathrm{a}}$ 、 $A_{\mathrm{f}}$ 参数下, 燃烧模式引起飞灰可燃物含量预测值的 最大差别在 2 倍左右, 在相同的燃烧模式下, 表 3 中的 $E_{\mathrm{a}} 、 A_{\mathrm{f}}$ 参数引起飞灰可燃物含量预测值最大差 别在 2.5 倍左右。

实际运行中, 烟气含氧量分布不均匀是引起飞 灰可燃物含量预测误差重要因素, 从图 6 可看出, $300.8 \mathrm{MW}$ 负荷下, 从炉膛 $\mathrm{A}$ 侧和 B 侧采集的灰样,
其飞灰可燃物含量差别较大, 这是炉内烟气含氧量 分布不均引起的, 沿烟道横截面的多点烟气采样分 析证实了这点; 如表 2, 在 180.6 MW 低负荷运行时, 炉膛出口过剩空气系数较高, 烟气含氧量较大, 大 颗粒在燃烧过程后期，颗粒被灰包围，由于灰分引 起的热退火抑制效应, 颗粒内部未燃烬碳真正能接 触到的氧气浓度会明显小于烟气中氧气浓度, 因此, 实际反应速率比预测值低, 这是造成预测值偏小的 主要原因。

\section{6 结论}

（1）传统炉膛分区段分传热计算方法经改进后 适合于空气分级燃烧, 通过引入燃烧模型, 能够预 测煤粉燃烬度及其在炉内热量的释放分布，计算量 较小, 适合工程应用。

（2）炉内局部烟气含氧量分布不均匀是引起模 型误差的主要因素; 对炉内氧量较高的低负荷运行 工况，飞灰可燃物预测值比实际测试值偏小较多， 主要原因是模型未能考虑颗粒燃烧后期灰分引起热 退火抑制效应。

(3) 燃烧模式对飞灰可燃物预测值有较大影 响, 炉内燃烧模式为等密度模式时, 预测值和测量 值较为接近。

（4）不同文献给出的煤焦表面反应频率因子和 活化能差别较大，对燃用煤种的频率因子和活化能 进行实际测试, 能进一步提高飞灰可燃物预测精度。

\section{参 考 文 献}

[1] 王小华, 陈宝康, 陈敏. 低 $\mathrm{NO}_{x}$ 燃烧器改造后飞灰可燃 物高原因分析及优化调整[J]. 热能动力工程, 2012, 27(3): 342-349.

WANG Xiaohua, CHEN Baokang, CHEN Min. Analysis of the causes for a high combustible content of the flying ash after a reconstruction of a low $\mathrm{NO}_{x}$ burner and its optimization adjustment[J]. Journal of Engineering for Thermal Energy and Power, 2012, 27(3): 342-349.

[2] 刘志江. 低氮燃烧器改造及其存在问题处理 [J]. 热力 发电, 2013, 42(3): 77-81.

LIU Zhijiang. Low $\mathrm{NO}_{x}$ Burner modification and the problems processing[J]. Thermal Power Generation, 2013, 42(3): 77-81.

[3] JAVIER P, INMACULADA A, ALAN W. Integration of CFD codes and advanced combustion models for quantitative burnout determination[J]. Fuel, 2007, 86(15): 
2283-2290.

[4] GAO H, MAJESKI A J. A method to target and correct sources of unburned carbon in coal-fired utility boilers[J]. Fuel, 2013, 108: 484-489.

[5] 北京锅炉厂. 锅炉机组热力计算标准方法[M]. 北京: 机械工业出版社, 1976.

Beijing Boiler Work, Ltd. Thermal calculation criterion for station bolier units[M]. Beijing: China Machine Press, 1976.

[6] 刘福国, 徐伟, 胡代军, 等. 消除大容量低 $\mathrm{NO}_{x}$ 切向燃 煤锅炉烟温偏差现场试验 [J]. 机械工程学报, 2013, 49(18): 159-166.

LIU Fuguo, XU Wei, HU Daijun, et al. Field tests elimination of the gas temperature deviation in large scale, advanced low $\mathrm{NO}_{x}$, tangentially fired pulverized coal boiler[J]. Journal of Mechanical Engineering, 2013, 49(18): 159-166.

[7] 张晓辉, 孙锐, 孙绍增, 等. 立体分级燃烧对 $\mathrm{NO}_{x}$ 排 放特性的影响 [J]. 机械工程学报, 2009, 45(2): 199-205. ZHANG Xiaohui, SUN Rui, SUN Shaozeng, et al. Effects of stereo-staged combustion technique on $\mathrm{NO}_{x}$ emmision charactisctics[J]. Journal of Mechanical Engineering, 2009, 45(2): 199-205.

[8] 肖琨, 高明, 乌晓江, 等. 空气分级低氮燃烧改造技术 对锅炉汽温特性影响研究[J]. 锅炉技术, 2012, 43(5): 62-65.

XIAO Kun, GAO Ming, WU Xiaojiang, et al. Effect of air staging low NO combustion technology on boi ier stearn temperature characteristic $[\mathrm{J}]$. Boiler Technology, 2012, 43(5): 62-65.

[9] 靳允立. 低 $\mathrm{NO}_{x}$ 燃烧技术改造对切园燃烧锅炉热量分 配的影响 $[\mathrm{J}]$. 中国电力, 2014, 47(3): 59-63.

JIN Yunli. Influence of low $\mathrm{NO}_{x}$ combustion technology modification on tangentially-fired boiler heat distribution [J]. Electric Power, 2014, 47(3): 59-63.

[10] 菲尔德 M A, 吉尔 D W, 摩根 B B, 等. 煤粉燃烧[M]. 北京: 水利电力出版社, 1989.

FIELD M A, GILL D W, MORGAN, B B, et al. Combustion of pulverized coal[M]. Beijing: Water Conservancy and Hydropower Press, 1989.

[11] FIELD M A. Rate of combustion of size-graded fractions of char from a low-rank coal between $1200 \mathrm{~K}$ and 2000K[J]. Combustion and Flame, 1969, 13(3): 237-252.

[12] HURT R, SUN J K, LUNDEN M. A kinetic model of carbon burnout in pulverized coal combustion[J].
Combustion and Flame, 1998, 113(1-2): 181-197.

[13] HURT R H, CALO J M. Semi-global intrinsic kinetics for char combustion modeling[J]. Combustion and Flame, 2001, 125(3): 1138-1149.

[14] STEPHENSON P. Computer modelling of the combined effects of plant conditions and coal quality on burnout in utility furnaces[J]. Fuel, 2007 (86): 2026-2031.

[15] 冯俊凯, 沈幼庭. 锅炉原理及计算 $[\mathrm{M}]$. 北京: 科学出 版社, 2003.

FENG Junkai, SHEN Youting. Theory and calculation of station boiler[M]. Beijing: Science Press, 2003.

[16] BIAGINI, TOGNOTTI E L. A generalized correlation for coal devolatilization kinetics at high temperature[J]. Fuel Processing Technology, 2014, 126: 513-520.

[17] BACKREEDY R I, HABIB R, JONES J M, et al. An extended coal combustion model[J]. Fuel, 1999, 78(14): 1745-1754.

[18] BAUM M M, STREET P J. Predicting the combustion behaviour of coal particles[J]. Combustion and Flame, 1971, 3(5): 231-243.

[19] FIELD M A. Measurements of the effect of rank on combustion rates of pulverized coal[J]. Combustion and Flame, 1970, 14(2): 237-248.

[20] JAVIER B, SANTIAGO J. Kinetic parameters for the oxidation of pulverised coalas measured from drop tube tests[J]. Combustion and Flame, 2005, 142(3): 210-222.

[21] KOUPRIANOV V I. Modeling of thermal characteristics for a furnace of a $500 \mathrm{MW}$ boiler fired with high-ash coal[J]. Energy, 2001, 26(9): 839-853.

[22] 容銮恩, 袁镇福, 刘志敏, 等. 电站锅炉原理[M]. 北 京: 中国电力出版社, 1997.

RONG Luanen, YUAN Zhenfu, LIU Zhimin, et al. The principle of power plant boiler[M]. Beijing: China Electric Power Press, 1997.

[23] 刘福国. 石横发电厂\#3 锅炉再热汽温度调整试验报告 [R]. 济南: 山东电力研究院, 2014.

LIU Fuguo. Test report on reheating temperature adjustment of \#3 boiler in Shi Heng power plant[R]. Jinan: Shandong Electric Power Research Institute, 2014.

[24] 国家标准化管理委员会. 电站锅炉性能试验规程 GB/T10184-2015[S]. 北京: 中国标准出版社, 2016. Standardization Administration of China. Performance test code for utility boiler GB/T10184-2015[S]. Beijing: Standard Press of China, 2016. 
[25] 刘福国, 刘科, 崔福兴, 等. 锅炉二次风挡板特性试 验数据处理方法与应用 $[\mathrm{J}]$. 热能动力工程, 2016 , 31(10): 115-121.

LIU Fuguo, LIU Ke, CUI Fuxing, et al. Data processing of air damper performance characteristics in boiler cold test and its application[J]. Journal of Engineering for Thermal Energy and Power, 2016, 31(10): 115-121.

[26] SMITH I W. The combustion rates of coal chars: A review[J]. Symposium on Combustion, 1982, 19(1):
1045-1065.

[27] KARLSTRÖM O, BRINK A, HERCOG J, et al. Kinetic combustion parameters for chars using the IFRF solid fuel data base[C]// Ifrf Members' Conference: Combustion and Sustainability: New Technologies, New Fuels, New Challenges, 2009.

作者简介: 刘福国, 男, 1969 年出生, 高级工程师。主要从事电厂锅炉 运行监测、诊断和优化方面的研究。

E-mail: lephico@163.com 